MS12-P9 Innovative protein crystallization screens

Fabrice GORREC ${ }^{1}$

1. MRC Laboratory of Molecular Biology, Francis Crick Avenue, Cambridge Biomedical Campus Cambridge, CB2 0QH, UK

email: fgorrec@mrc-lmb.cam.ac.uk

At the MRC-LMB, despite using a set of commercially available conditions considered as very large $(\geq 1440$ conditions), the yield of experiments containing protein crystals with sufficient quality to solve structures is regularly below $1 \%$. In addition to sample properties, an underlying reason for such low yield is the very large number of combinations of variables associated with protein crystallization.

We argue that our approach to initial crystallization screening is yet under-sampled and hence an even larger set of conditions should be preferred whenever possible. Protein crystallization screen formulations should evolve in parallel with the increasing complexity of the samples and the technical difficulties encountered during the process of structure determination. Notably, demands of cryo-crystallography, as well as solutions to the phase problem should be taken into account more. In this context, developed the Pi and MORPHEUS approaches to formulation and the corresponding screens.

The formulation of a $\mathrm{Pi}$ screen is derived from the incomplete factorial approach. Combinations of 3 reagents are produced following the modular distribution of a given set of up to 36 stock solutions. Maximally diverse conditions can be produced by taking into account the properties and the concentrations of the chemicals used to formulate a 96-condition screen. The Pi sampling method is intended to help laboratories on a day-to-day basis to test new crystallization screen formulations based on the properties of the macromolecules investigated.

A MORPHEUS screen (also consisting of 96 conditions) integrates several innovative approaches developed elsewhere, such as mixes of potential ligands, convenient buffer systems and precipitant mixes that also act as cryoprotectants. Molecules frequently observed in the PDB to co-crystallize with proteins have been selected to formulate MORPHEUS I and II. The aim was to increase yield of quality crystals with molecules acting as protein stabilizers, crystal packing bridges, or any other role beneficial to protein crystallization.

Increasing the initial yield of quality crystals is key for the determination of novel protein structures by X-ray diffraction. Ideally, demanding optimizations and issues with reproducibility will be bypassed.

Keywords: crystallography; proteins; crystallization; screen; formulation; additives

\section{MS12-P10 Protein surface modifying agents in protein crystallization}

Jindřich Hašek ${ }^{1}$, Tereza Skálová ${ }^{1}$, Petr Kolenko ${ }^{1}$, Jarmila Dušková ${ }^{1}$, Tomáš Koval ${ }^{1}$, Karla Fejfarová ${ }^{1}$, Jan Stránský ${ }^{1}$, Jan Dohnálek $^{1}$

1. Institute of Biotechnology, Academy of Sciences of the CR, Vídeňská 1083, 14220 Prague 4, Czech Republic

email: hasekjh@seznam.cz

Uniform stacking of protein molecules into the growing crystal requires a dominance of a single protein-protein adhesion mode. Existence of mutually incompatible protein-protein adhesion modes in the same crystal leads to stacking faults, to low quality crystals, or to crystallization failure. The substances balancing kinetics of the protein-protein adhesion in different adhesion modes are called "Protein Surface Active Molecules" (PSAM) [1]. The PSAM binding selectively and temporarily to the protein surface compete with protein-protein adhesion modes, or sometimes can form the protein-protein crosslinks built-in permanently into the growing crystals.

The theory of PSAM describes protein crystallization as a regular deposition of short-life protein-PSAM adducts and stresses a role of these "sticking molecules" with highly specific adhesion to different protein-surface patches active in crystallization processes. The PSAM-protein adducts decompose after the protein is fixed on crystal surface or when the other protein adducts try fix in correct position and orientation nearby on the growing crystal surface. Proper choice of PSAM eliminates deposition of protein molecules in the crystallographically incompatible protein-protein adhesion modes. It is decisive for a success of protein crystallization in many cases.

The concept of crystallization as a regular deposition of naked protein molecules on the growing crystal surface is here replaced by the concept of regular deposition of the PSAM-protein adducts. It is more difficult for imagination, but provides explanations of many enigmatic crystallization phenomena. It increases the number of crystallizable proteins, allows preparation of crystals with protein-protein complexes, and leads to higher quality diffraction data by minimizing probability of stacking faults in crystal. It explains why combination of several "successful additives" can lead to crystallization failure. It allows also a preparation of polymorphs allowing study of protein conformation in different environments with different $\mathrm{pH}$, different water content, etc. One can also choose the polymorph ensuring the highest accuracy of structure determination.

The study supported by ERDF CZ.1.05/1.1.00/02.0109 BIOCEV, LG14009, CSF 15-15181S, MSMT EE2.3.30.29, SGS13/219/OHK4/3T/14

[1] Hašek, J. (2006) Z. Kristallogr. 23, 613-619; Hašek, J. (2011) J. Synchr.Radiation 18, 50-52; Hašek, J. et al (2011) Z. Kristallogr. 28, 475-480.

Keywords: Keywords: protein crystallization, protein-protein adhesion, protein surface, hydrophilic polymers 